\title{
Physician Burnout in Canada: Challenges and Possible Future Interventions
}

\author{
Karuna Manchanda ${ }^{1}$, MBBS, RHDS \\ ${ }^{1}$ School of Health Administration, Dalhousie University \\ DOI: https://doi.org/10.15273/hpj.v1i2.10588
}

\begin{abstract}
Background: Physicians are the backbone of the health-care industry, and the state of their wellbeing significantly influences the productivity, financial conditions and overall performance of the health care system. The objectives of this literature review are to highlight the challenges posed by physician burnout to the Canadian health care system and suggest possible recommendations to reduce this burnout. Methods: Database searches were conducted to study the effects of physician burnout on the health care system. Websites of organizations like the Canadian Medical Association, Canadian Medical Protective Association, Canadian Collaborative Centre for Physician Resources and Medical Council of Canada were searched. Research papers from Canada, the United States, Europe and Hong Kong were assessed to support the existence of burnout among physicians in Canada and globally. Results: Burnout affects the Canadian health care system in terms of estimated cost, patient safety, productivity, performance, COVID-19 pandemic response and future medical workforce supply. It was found that the level of physician burnout is increasing notably among the Canadian workforce, with the COVID-19 pandemic adding more stress to the situation. The costs of burnout rendered to the health care system are continually increasing each year in Canada. Many physicians across the world are considering retiring early, while others are considering quitting the profession altogether, adding to the pressure already imposed on the physicians remaining in the system. Recommendations to reduce burnout among physicians from the literature were studied as part of this review. Conclusion: Physician burnout has several negative impacts on the health care system. This literature review provides a concise overview of the major challenges posed by burnout among Canadian physicians. It will help contribute to plans and strategies to reduce physician burnout in the future.
\end{abstract}


Physician Burnout in Canada: Challenges and
Possible Future Interventions

Introduction

Burnout among health professionals is not only common in Canada but has become more of a global issue in recent years (Dewa, Jacobs, et al., 2014). Interest in studying this phenomenon is increasing because of its heavy impact on health care outcomes and the dire need to overcome associated challenges (Shanafelt et al., 2017). Physician burnout is characterized by three symptoms: exhaustion, depersonalization and lack of efficacy (low sense of personal accomplishment; Drummond, 2015). Burnout also claims its victims in a personal manner with regard to higher risk of alcohol abuse and dependence and an associated increased risk of suicidal ideation (Dyrbye et al., 2008). The numerous stresses of the job, along with the typical ideology of not exhibiting fatigue, contribute to burnout becoming an inevitable outcome for all physicians (Boyle, 2018). There have been many studies conducted in the past to better understand the causes of physician burnout in Canada (Dewa, Jacobs, et al., 2014). Over the years, Canadians have been experiencing increasing wait times to access medical services, and it has been a major contributing factor to the burden on the health care system (Dewa, Jacobs, et al., 2014). The purpose of this literature review is to outline the major challenges that physician burnout poses to the health care system in Canada as well as to offer possible recommendations to reduce this burnout.

\section{Methods}

For the purposes of this rapid review, a systematic search of both grey and published literature was performed. The databases that were searched included PubMed, MEDLINE, Google Scholar and PsycInfo. Websites of organizations like the Canadian Medical Association (CMA), Canadian Medical Protective Association, Canadian Collaborative Centre for Physician Resources (C3PR) and Medical
Council of Canada were also searched. Examples of keywords used to conduct the search included "physician," "burnout," "patient safety," and "COVID-19." Only papers written in English and focusing on physician burnout from a national perspective were included. Papers regarding burnout in other medical professions (e.g., nursing) were excluded.

The search strategy conducted for this review produced 19 papers that met the inclusion criteria. Eleven of these research papers were from Europe and the United States, and the remaining eight were from Canada. Standard rapid review procedures (Dobbins, 2017) were followed with respect to database searches and article translations. Recommendations were extracted from the literature and personal recommendations were also articulated for the purposes of this paper.

\section{Results}

\section{Estimated Cost of Physician Burnout in Canada}

A study conducted by Dewa, Jacobs et al. (2014) helps in establishing an estimate of the cost of burnout associated with Canadian physicians. It utilized an economic model and was supported by data from National Physician Surveys. Physician data was analyzed on the basis of their specialty, weekly work hours, patient load per hour and patient visits per week. The cost of early retirement was based on the annual loss of physician revenue and the result was an estimated loss of $\$ 118.53$ million (2010 CAD\$) per year from family physicians retiring early. Loss of revenue due to early retirement of surgeons was estimated to be around \$33.68 million, and from other specialists, $\$ 32.96$ million. On the other hand, the cost of reduction in clinical hours was calculated by estimating the reduction in visits per week using the difference in weekly visits between dissatisfied and not dissatisfied physicians. An estimated $\$ 6.8$ million was projected to be lost from family physicians reducing their clinical hours. The total cost of burnout for all physicians in Canada was estimated to be $\$ 213.1$ million, presented as a 
future value of service reductions (Dewa, Jacobs, et al., 2014). The future value is a discounted value calculated to estimate the time value of dollars in the future (Vo, 2019). This estimate is conservative because it is calculated as the difference in costs for physicians who experienced burnout versus the costs if those physicians did not experience burnout (Dewa, Jacobs, et al., 2014).

\section{Relationship Between Physician Productivity and Burnout}

A paper by Dewa, Loong, et al. (2014) includes a systematic literature review of five studies conducted in the US, China, Hong Kong, the Netherlands, the United Kingdom and 11 other European countries. Global reports suggest that burnout results in low job satisfaction, deteriorating mental health and poor quality of care (Dewa, Loong, et al., 2014). All these outcomes impact the level of productivity in the workplace. Burnout was measured using the Maslach Burnout Inventory-Human Services Survey (Maslach \& Jackson, 1981). Productivity was measured and outcomes discussed using the following four parameters: sick leave, intent to change jobs, intent to quit practising medicine, and work ability (Dewa, Loong, et al., 2014). With regard to the first outcome of sick leave, the study from the European countries suggested that all three symptoms of burnout (exhaustion, depersonalization and lack of efficacy) are significantly associated with more use of sick days (Soler et al., 2008). In contrast, Siu et al. (2012) reported no statistically significant differences in the average sick leave days in the past year for public hospital physicians with and without high burnout scores. There are conflicting results reported for this parameter because productivity measures and working styles are different for each individual (Dewa, Loong, et al., 2014). Secondly, the intent to change jobs was strongly associated with high burnout scores. The intent to quit practising medicine was the third outcome and qualified as a strong indicator of burnout among physicians. The fourth outcome was of work ability and was defined by Ruitenburg et al. (2012) as "the degree to which a worker is physically and mentally able to cope with the demands of the work" (p. 2). Focusing on Canada, the CMA's annual Physician Resource Questionnaire in 2002 revealed that physicians worked an average of 53.8 hours per week, excluding oncall activities, compared with 53.4 hours in 2001 and 52.9 hours in 2000 (Martin, 2002). The 2002 survey was mailed to a random sample of 7,693 doctors, and the response rate was $38 \%$. Of these physicians, $9 \%$ reported reducing the scope of their practice in the last two years to reduce the workload and increase productivity (Martin, 2002). Several of these physicians reported being dissatisfied with their lifestyle, with an increasing patient load and less time to spend with their families (Martin, 2002). The responses signified a rising need to incorporate measures to reduce burnout among these physicians.

\section{Effect of Burnout on Patient Safety}

There are a number of studies conducted across North America and several surveys done in Canada that suggest burnout and patient safety have a negative relationship (Salyers et al., 2017). The meta-analysis conducted by Salyers et al. suggests that there is a statistically significant decrease in patient safety and quality of care with increasing burnout among physicians. The meta-analysis of the relationship between burnout and patient safety included 40 independent samples and resulted in a significant negative relationship $(r=-0.23)$, with a confidence interval of $95 \%$ ranging from -0.28 to -0.17 . It was also noted that emotional exhaustion is likely the most critical element of burnout to address (Salyers et al., 2017). According to the Canadian Medical Protective Association, burned-out physicians reported taking shortcuts, failed to follow established procedures, did not answer patient questions, did not discuss treatment options, and made treatment errors that could not be attributed to a lack of knowledge (The Canadian Medical Protective Association, 2018a). These shortcomings translated to poor quality of care along with longer recovery times among patients, which, in turn, increased waiting times. 
When looked at from the perspective of the building blocks of health care systems, burnout is responsible for negatively impacting service delivery, health care workforce and financing (World Health Organization, 2010).

\section{Impact of Burnout on Health System Performance}

According to Sinsky et al. (2017), physicians are exiting their careers in medicine faster than they enter; their intentions to withdraw are highly correlated with burnout with an odds ratio of 5.8. This suggests that there will be a severe shortage of practising physicians in the near future. This negative impact on the health system performance is directly related to burnout and can be averted if due attention is paid to it. The prevalence rate of burnout in the United States is only 2.6 times the rate of that in Canada, even though the physician workforce in the United States is 12 times bigger (Sinsky et al., 2017). A survey was conducted in the United States in 2014 (Sinsky et al., 2017). Out of the 6,695 physicians in clinical practice in the US at the time of the survey, $19.8 \%$ of those who responded reported it was likely or definite that they would reduce clinical work hours in the next 12 months, while $26.6 \%$ indicated it was likely or definite that they would leave their current practice in the next two years (Sinsky et al., 2017). This demonstrates the prevalence of burnout in the United States causing physicians to either quit the profession or reduce work hours. It is important to note that even with Canada's smaller physician workforce, the burnout rates are much greater than expected (Sinsky et al., 2017). This burnout is increasing because physicians are physically present but psychologically withdrawn, the result being major effects on patient health outcomes, efficiency and productivity (Olson, 2017). All these factors together constitute the overall health system performance. In a survey conducted by the CMA in 2017, it was found that $74 \%$ of family/general physicians were partially or completely closed to accepting new patients (CMA, 2017). An interpretation of this result suggests that patient load is constantly increasing in Canada, adding to the rising levels of burnout among Canadian physicians and the deteriorating quality of health-care service delivery.

\section{Physician Work Hours in Canada}

As seen in Table 1, physician work hours in Canada have been relatively consistent over the years (CMA, 2017). However, Canadian Medical Protective Association data shows that over the past five years, college and hospital complaints have risen by more than $20 \%$, and burnout may be associated with this increase (The Canadian Medical Protective Association, 2018b). Moreover, the CMA conducted an online survey in 2017 with almost 3,000 physicians and residents. According to its findings, one in three Canadian physicians screened positive for depression (Boyle, 2018), and a significantly higher number of residents reported burnout and depression than physicians (CMA, 2018). The CMA President also raises the issue of prevailing stigma in the field, which prevents many health professionals from seeking help (Boyle, 2018). In order to reduce burnout in Canada as well as globally, the first step is to remove these stigmas from the health care environment. Despite being mentally, physically and emotionally exhausted, physicians continue to work, resulting in more medical errors that consequently increase health care costs.

\section{Effect of the COVID-19 Pandemic on Physician Burnout}

In light of the COVID-19 pandemic, the situation of burnout among Canadian physicians has become even worse. There is a national shortage of emergency medical professionals in Canada, and on top of that, $86 \%$ of those currently practising emergency medicine experience burnout (Chochinov \& Lim, 2020). The pandemic superimposed on the already dysfunctional emergency departments of Canada is proving to be one of the biggest crises the health industry needs to combat. Moreover, the pandemic is exhausting physicians to the brink of considering suicide, and access to toxic medication is increasing the risk (Gulati \& Kelly, 2020). There are several instances in which a physician may suffer from moral injuries: 
Table 1

Average Hours Worked per Week by Canadian Physicians, 1997-2017

cma.ca | amc.ca

Average hours worked per week by physicians, 1997-2017

\begin{tabular}{|c|c|c|c|c|c|c|c|c|c|c|c|c|}
\hline & 1997 & 1998 & 1999 & 2000 & 2001 & 2002 & 2003 & 2004 & 2007 & 2010 & $2014^{*}$ & $2017^{*}$ \\
\hline Activity & $n=3350$ & $n=3285$ & $n=3050$ & $n=2734$ & $n=3123$ & $n=2763$ & $n=2173$ & $n=20332$ & $\begin{array}{l}n=18061 \\
N=54214^{\star \star}\end{array}$ & $\begin{array}{l}n=11069 \\
N=60814^{\star \star}\end{array}$ & $\begin{array}{l}n=9423 \\
N=66241^{\star \star}\end{array}$ & $\begin{array}{l}n=6601 \\
N=69433^{\star *}\end{array}$ \\
\hline Direct patient care & 35.6 & 36.2 & 35.9 & 35.0 & 35.0 & 35.6 & 35.4 & 34.0 & 33.3 & 32.7 & 31.0 & 33.0 \\
\hline Health facility committees & 1.5 & 1.3 & 1.4 & 1.2 & 1.3 & 1.2 & 1.2 & 0.8 & 1.0 & 0.8 & 0.9 & 0.9 \\
\hline Managing practice & 2.2 & 2.0 & 2.4 & 2.3 & 2.2 & 2.5 & 2.2 & 1.5 & 1.6 & 1.6 & 1.5 & 1.4 \\
\hline Other indirect patient care & 4.5 & 4.5 & 4.9 & 5.1 & 4.8 & 5.0 & 5.2 & 5.3 & 5.9 & 6.1 & 6.2 & 6.9 \\
\hline Research & 1.6 & 1.6 & 1.6 & 1.6 & 1.8 & 1.7 & 1.5 & 1.5 & 1.6 & 1.6 & 1.3 & 1.2 \\
\hline Administration & 2.1 & 2.2 & 2.3 & 2.2 & 2.3 & 2.0 & 1.8 & 2.2 & 2.2 & 2.5 & 2.4 & 2.3 \\
\hline $\begin{array}{l}\text { Teaching/educating without } \\
\text { direct patient care }\end{array}$ & 1.6 & 1.5 & 1.4 & 1.4 & 1.6 & 1.6 & 1.4 & 1.2 & 1.5 & 1.6 & 1.6 & 1.4 \\
\hline $\begin{array}{l}\text { Continuing medical } \\
\text { education }\end{array}$ & 2.9 & 2.9 & 3.0 & 3.0 & 3.1 & 3.2 & 3.0 & 3.1 & 3.2 & 3.2 & 3.0 & 2.6 \\
\hline Other activities & 1.2 & 1.1 & 1.2 & 1.1 & 1.2 & 1.1 & 1.2 & 1.1 & 1.2 & 1.3 & 0.8 & 0.9 \\
\hline Total & 53.2 & 53.4 & 54.1 & 52.9 & 53.4 & 53.8 & 52.9 & 50.7 & 51.7 & 51.4 & 48.7 & 50.5 \\
\hline \multicolumn{13}{|c|}{ 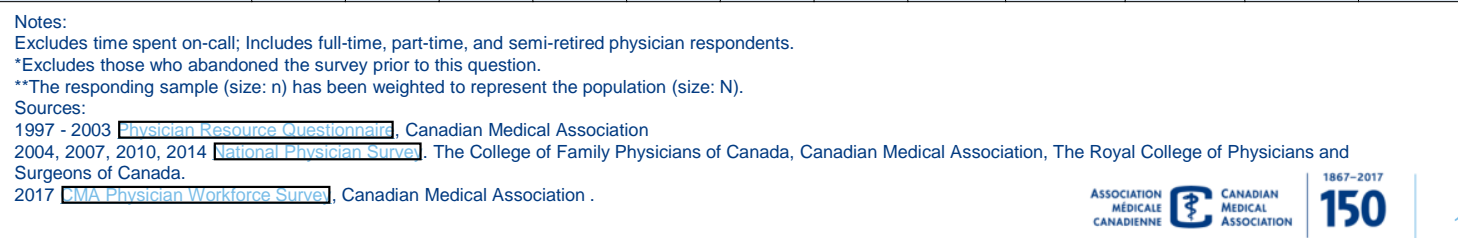 } \\
\hline
\end{tabular}

Note. From Average hours worked per week by physicians, 1997-2017, by CMA, 2017.

situations where there are ethical conflicts regarding which patients to admit (Parker \& Mirzaali, 2020) or where physicians are professionally isolated due to reduced access to peer groups and social distancing protocols (Gulati \& Kelly, 2020). Overall, physician burnout has never been worse globally and there is a growing need to provide support to these physicians. Some suggestions made by Gulati and Kelly include hospitals being made to establish a COVID-19 Clinical Ethics Committee (Gulati \& Kelly, 2020). Physicians should be able and even required to have meetings with this committee on short notice in order to be supported in the clinical decisions they make (Gulati \& Kelly, 2020). This would reduce the burden of responsibility on individual physicians and relieve some symptoms of burnout. Secondly, in accordance with maintaining social distancing, physician peer groups should hold regular virtual meetings that allow physicians to discuss their problems. Such informal sessions can prove to be of utmost significance in fighting the challenges associated with professional isolation (Gulati \& Kelly, 2020).

\section{Recommendations to Reduce Physician Burnout from the Literature}

Burnout and depression have been identified in almost half of all practising physicians in Canada (Boyle, 2018). In a study conducted by Kuhn and Flanagan (2017), it is suggested that, due to increasing work demands, many physicians feel there are fewer opportunities to appreciate the patient-doctor relationship that historically was a privilege of medicine. A few steps outlined in this paper to overcome burnout in Canadian physicians include promoting mindfulness and teamwork for trainees and physicians, developing practice models that preserve physician work control, supporting manageable patient panel sizes, promoting career opportunities and advancements for physicians, and prioritizing self-care as part of medical professionalism (Kuhn \& Flanagan, 2017). It is essential to the well-being of physicians to engage in self-care 
practices, beginning with reporting and seeking help for stress associated with workload. Institutional metrics should be including a greater focus on physician satisfaction and wellbeing in their reports and research agendas. Physicians should be given sufficient control over their work hours and the length of patient visits to maintain a healthy work-life balance. Issues like added stress from maintaining electronic health records should be investigated, and staffing in such clinics should be increased to battle the extra workload placed on physicians (Kuhn \& Flanagan, 2017). While medical boards and credentialing services focus on diagnosing depression among medical professionals (Kuhn \& Flanagan, 2017), they should be working on encouraging physicians to seek help without the concerns of facing licensure limitations. A more result-oriented approach can include the formation of a committee focused on physician wellness, with its sole purpose being mobilization of more resources to educate medical professionals about burnout and suggest strategies to combat the same.

While many physician-directed interventions are being suggested, a systematic review and meta-analysis is conducted by De Simone et al. (2019) to evaluate the effectiveness of organizational strategies to reduce physician burnout. There are several issues related to work environment and organizational culture that affect physicians in a negative manner. Modifying staffing schedules in order to reduce physician shift hours (Garland et al., 2012), developing targeted quality improvement projects (Linzer et al., 2015), implementing changes in workflow, and designating periods of protected time slots where physicians and residents are required to de-stress (Shea et al., 2014) are some of the organizational interventions discussed in the systematic review. Organization-directed interventions were associated with a medium reduction in burnout score (Standardized Mean Difference of -0.446 with a 95\% Confidence Interval ranging between -0.619 and -0.274 ), while physician-directed interventions were associated with a small reduction in burnout score (Standardized Mean Difference of -0.178 with a 95\% Confidence Interval ranging between -0.332 and -0.035 ; De Simone et al., 2019). Evidently, organization-directed interventions had a stronger impact on reducing burnout among physicians, since the strategies were applied on a larger scale and included improving the basic organizational workflow. To further discuss management of burnout in physicians, Panagioti et al. (2017) conducted a meta-analysis on randomized clinical trials and controlled before-after studies of interventions targeting burnout in physicians. This study further supports the results drawn from the study conducted by De Simone et al. (2019). It is stated that organization-directed interventions are more effective in diminishing the symptoms of burnout in physicians; however, such interventions are relatively rare (Panagioti et al., 2017). A total of 19 studies were analyzed by Panagioti et al. to assess effectiveness of controlled interventions in addressing physician burnout. Forty percent of these studies focused on organization-directed interventions like workload evaluations, discussion meetings to enhance teamwork, and structural changes in the organization to support physician wellness (Panagioti et al., 2017). Moreover, there were differences in burnout outcomes for physicians working in different health-care settings like primary or secondary care. Interventions in primary care had small to medium reductions in burnout, whereas interventions in secondary care were associated with small significant reductions in burnout. Thus, the overall results were similar to the ones revealed by De Simone et al. (2019), which signifies the need to strengthen organization-directed interventions in health-care settings.

\section{Author's Recommendations}

The Canadian health care system is considered one of the best in the world (Ireland, 2021), but it has some shortcomings. Due to shortage of medical staff throughout the country, burnout among medical professionals is continuously increasing. When examined from a deeper perspective, the root of the 
problem lies in the scarcity of medical infrastructure. There are very few teaching facilities for medicine in the country. Thus, many medical students take up their careers in other countries like the United States (Slade, 2014). This results in a loss of potential medical workers in the Canadian health care system and causes a shortage of supply (Sinsky et al., 2017). A potential recommendation regarding this issue is for governments to increase the investment in more medical facilities and teaching institutes in Canada. That, in turn, will produce more future medical professionals who intend to work in this country. It will also help in reducing the workload of existing physicians and discouraging them from retiring early or reducing their current work hours. Furthermore, it was observed that the concept of electronic health records is relatively new in Canada and has become a source of major burnout among physicians. The use of technology requires a fair amount of experience, and many elderly physicians are facing unnecessary stress due to lack of technology skills (Kuhn \& Flanagan, 2017). This problem can be overcome by introducing specialists like medical transcriptionists who are professionally trained to maintain electronic health records. The excess of workload related to this particular task can be removed from physicians' daily duties and can aid in reducing burnout.

Moreover, the problem of physician shortage in Canada can also be attributed to the cumbersome process that internationally trained physicians must navigate in order to join the Canadian workforce. As discussed previously, there is a huge outflow of potential medical workers due to lack of teaching facilities in Canada. To combat this outflow, a potential solution includes bringing physicians from other countries into Canada. However, the process for international medical graduates (IMGs) to enter Canada and successfully practise medicine is particularly cumbersome. They are required to appear for several examinations including the Medical Council of Canada Qualifying Examination and complete their medical residency again, even if they have already done so in their home country (Medical Council of
Canada, n.d.). This poses as a huge barrier for foreign physicians who are considering choosing Canada to build their careers. The only way to overcome this barrier is by re-evaluating the medical entrance procedure and somewhat modifying it to resemble the British system. In the UK, IMGs can take the Professional and Linguistic Assessments Board tests, as well as the Membership of the Royal Colleges of Physicians of the United Kingdom test, and start a training job in the National Health Service of the United Kingdom (Ivan, n.d.). This gives IMGs a way to support themselves in a new country while working toward building their medical career without having to pursue a residency. After working the training job for two to three years, they can apply for their specialist/GP registration and continue practising medicine (Ivan, n.d.). If a system like this is implemented in Canada, many IMGs will start choosing Canada to build their medical career. There will be a consistent inflow of new physicians, further reducing the problem of burnout in the country.

Several supporting studies and surveys have been conducted in the past to better understand the problem of physician shortage and burnout in Canada. In the 1970s, Canada had a growing physician population, which resulted in a physician-to-population ratio of 1.91/1,000 in 1993 (CMA, 2013). However, the federal government realized that there was a surplus of physicians, and subsequently implemented policies to control this growth (Malko \& Huckfeldt, 2017). The policies caused the net inflow of physicians into the physician pool to drop from 1,040 physicians per year in the period of 1990-1993 to 313 physicians in the period of 1994-2000 (Chan, 2002). The government then made efforts to increase the physician population. However, despite the efforts, the physician-population ratio in Canada was 2.24/1,000 in 2014, which ranked Canada 28th among 34 OECD countries (C3PR, 2013). To further aggravate the problem, the current physician pool as well as the general population in Canada are aging: forty-one percent of Canada's physicians were aged 55 or older in 2013 (C3PR, 2013). Additionally, the aging population of the country comprises Canadians 
aged 65 and older who consume $45 \%$ of the total health care budget (Canadian Institute for Health Information, 2014). Due to shortage of new physicians, the existing physicians are burdened with the health issues of the aging population. These numbers suggest that there is a dire need to expand the physician population of Canada in order to combat the growing shortage in the workforce.

\section{Discussion}

Physician burnout in Canada is a problem that cannot be overcome easily. It not only reduces efficiency of the medical staff but also increases medical errors, thus reducing patient safety. The inability to physically withdraw from patients pushes physicians to psychologically withdraw from them, resulting in worsening health outcomes for the patients (Olson, 2017). Following this, there is an increase in recovery times, further increasing the wait time problem in Canada. Further, since many physicians have been leaving the profession or reducing their work hours, the loss of revenue from physicians has massively increased over the years (Martin, 2002). Over the years, the percentage of physicians accepting new patients has been dropping; it is becoming a hassle for patients to look for a general physician (CMA, 2017), and this reduces the quality of basic health service delivery in the country. Currently, a very small amount of research papers are available that focus on physician burnout specifically in Canada. This issue needs to be thoroughly investigated on a national level in order to devise further strategies and resolutions in the future.

\section{Concluding Remarks}

The problem of physician burnout has existed for a long time globally, and its consequences have been impacting health care service delivery. It will require a great deal of effort and some major organizational changes to fight this epidemic of physician burnout. Removing the stigma that prevents physicians from discussing their stresses, implementing strategies to reschedule long shifts, and reducing the number of patients per physician are some measures that can help in reducing physician fatigue. Organizational change will aid physicians in becoming more direct about seeking help and becoming less burdened (De Simone et al., 2019). It is also important to note that if the government does not take steps to increase medical infrastructure or, alternatively, increase the inflow of physicians, Canada will face a severe shortage of medical workers in the future.

\section{References}

Boyle, T. (2018, October 10). Canadian doctors are suffering from burnout at an 'alarming' rate, survey finds. The Star. https://www.thestar.com/news/gta/2 018/10/10/md-burnout-an-alarmingproblem-cma-president-says.html

Canadian Collaborative Centre for Physician Resources. (2013). Canadian physician resources - 2013 basic facts.

Canadian Institute for Health Information. (2014). National health expenditure trends, 1975 to 2014.

https://publications.gc.ca/collections/c ollection_2015/icis-cihi/H118-2-2014eng.pdf

Canadian Medical Association. (2013). Physicians per 100,000 population by province/territory, Canada, 1986-2015. https://www.cma.ca/sites/default/files /pdf/Physician\%20Data/12Phys_per_pop.pdf

Canadian Medical Association. (2017). Average hours worked per week by physicians, 1997-2017. https://www.cma.ca/sites/default/files /pdf/Physician\%20Data/34-

TrendData-e.pdf

Canadian Medical Association. (2018). CMA

National Physician Health Survey: A national snapshot. https://www.cma.ca/sites/default/files /2018-11/nph-survey-e.pdf

Chan, B. T. B. (2002, June). From perceived surplus to perceived shortage: What 
happened to Canada's physician workforce in the 1990s? Canadian Institute for Health Information. https://secure.cihi.ca/free_products/ch anjun02.pdf

Chochinov, A., \& Lim, R. (2020). On the brink of burnout: COVID-19 and the ER. Canadian Association of Emergency Physicians. https://caep.ca/wpcontent/uploads/2020/03/CAEPWellness-OpEd_Final_Clean.pdf

De Simone, S., Vargas, M., \& Servillo, G. (2019). Organizational strategies to reduce physician burnout: A systematic review and meta-analysis. Aging Clinical and Experimental Research, 33(4), 883-894. https://doi.org/10.1007/s40520-01901368-3

Dewa, C. S., Jacobs, P., Thanh, N. X., \& Loong, D. (2014). An estimate of the cost of burnout on early retirement and reduction in clinical hours of practicing physicians in Canada. BMC Health Services Research, 14, Article 254. https://doi.org/10.1186/1472-696314-254

Dewa, C. S., Loong, D., Bonato, S., Thanh, N. X., \& Jacobs, P. (2014). How does burnout affect physician productivity? A systematic literature review. $B M C$ Health Services Research, 14, Article 325. https://doi.org/10.1186/14726963-14-325

Dobbins, M. (2017, July 12). Rapid review guidebook: Steps for conducting a rapid review. National Collaborating Centre for Methods and Tools. https://www.nccmt.ca/uploads/media /media/0001/01/a816af720e4d587e1 3da6bb307df8c907a5dff9a.pdf

Drummond, D. (2015). Physician burnout: Its origin, symptoms, and five main causes. Family Practice Management, 22(5), 4247.

https://www.aafp.org/fpm/2015/0900 /p42.html

Dyrbye, L. N., Thomas, M. R., Massie, F. S., Power, D. V., Eacker, A., Harper, W., Durning, S., Moutier, C., Szydlo, D. W.,
Novotny, P. J., Sloan, J. A., \& Shanafelt, T. D. (2008). Burnout and suicidal ideation among U.S. medical students. Annals of Internal Medicine, 149(5), 334-341. https://doi.org/10.7326/0003-4819149-5-200809020-00008

Garland, A., Roberts, D., \& Graff, L. (2012). Twenty-four-hour intensivist presence: A pilot study of effects on intensive care unit patients, families, doctors, and nurses. American Journal of Respiratory and Critical Care Medicine, 185(7), 738743.

https://doi.org/10.1164/rccm.20110917340C

Gulati, G., \& Kelly, B. D. (2020). Physician suicide and the COVID-19 pandemic. Occupational Medicine, 70(7), 514. https://doi.org/10.1093/occmed/ kqaa104

Ireland, S. (2021, April 27). Revealed: Countries with the best health care systems, 2021. CEOWORLD Magazine. https://ceoworld.biz/2021/04/27/rev ealed-countries-with-the-best-healthcare-systems-2021/

Ivan, I. (n.d.). Post graduation pathways in the UK for IMGs. Road to UK. https://www.roadtouk.com/postgradu ation/postgraduation-pathways/

Kuhn, C.M., \& Flanagan, E.M. (2017). Self-care as a professional imperative: Physician burnout, depression, and suicide. Canadian Journal of Anesthesia, 64, 158-168. https://doi.org/10.1007/s12630-0160781-0

Linzer, M., Poplau, S., Grossman, E., Varkey, A., Yale, S., Williams, E., Hicks, L., Brown, R. L., Wallock, J., Kohnhorst, D., \& Barbouche, M. (2015). A cluster randomized trial of interventions to improve work conditions and clinician burnout in primary care: Results from the Healthy Workplace (HWP) Study. Journal of General Internal Medicine, 30(8), 1105-1111. https://doi.org/10.1007/s11606-0153235-4 
Malko, A. V., \& Huckfeldt, V. (2017). Physician shortage in Canada: A review of contributing factors. Global Journal of Health Science, 9(9), 68-80. https://doi.org/10.5539/gjhs.v9n9p68

Martin, S. (2002). More hours, more tired, more to do: Results from the CMA's 2002 Physician Resource Questionnaire. CMAJ : Canadian Medical Association Journal, 167(5), 521-522. https://www.cmaj.ca/content/167/5/5 21.1

Maslach, C., \& Jackson, S. E. (1981). The measurement of experienced burnout. Journal of Organizational Behavior, 2(2), 99-113.

https://doi.org/10.1002/job.40300202 05

Medical Council of Canada. (n.d.). Medical Council of Canada Qualifying Examination Part I. https://mcc.ca/examinations/mccqepart-i/

Olson, K. D. (2017). Physician burnout-A leading indicator of health system performance? Mayo Clinic Proceedings, 92(11), 1608-1611.

https://doi.org/10.1016/j.mayocp.201 7.09.008

Panagioti, M., Panagopoulou, E., Bower, P., Lewith, G., Kontopantelis, E., ChewGraham, C., Dawson, S., van Marwijk, H., Geraghty, K., \& Esmail, A. (2017). Controlled interventions to reduce burnout in physicians: A systemic review and meta-analysis. JAMA Internal Medicine, 177(2), 195-205. https://doi.org/10.1001/jamainternme d.2016.7674

Parker, J., \& Mirzaali, M. (2020, March 16). The moral cost of coronavirus. Journal of Medical Ethics Blog.

https://blogs.bmj.com/medicalethics $/ 2020 / 03 / 16 /$ the-moral-cost-ofcoronavirus/

Ruitenburg, M. M., Frings-Dresen, M. H. W. \& Sluiter, J. K. (2012). The prevalence of common mental disorders among hospital physicians and their association with self-reported work ability: A cross-sectional study. BMC Health Services Research, 12, Article 292. https://doi.org/10.1186/14726963-12-292

Salyers, M. P., Bonfils, K. A., Luther, L., Firmin, R. L., White, D. A., Adams, E. L., \& Rollins, A. L. (2017). The relationship between professional burnout and quality and safety in healthcare: A metaanalysis. Journal of General Internal Medicine 32(4), 475-482. https://doi.org/10.1007/s11606-0163886-9

Shanafelt, T. D., Lightner, D. J., Conley, C. R., Petrou, S. P., Richardson, J. W., Schroeder, P. J., Brown, W. A. (2017). An organizational model to assist individual physicians, scientists, and senior health care administrators with personal and professional needs. Mayo Clinic Proceedings, 92(11), 1688-1696. https://doi.org/10.1016/j.mayocp.201 7.08.020

Shea, J. A., Bellini, L. M., Dinges, D. F., Curtis, M. L., Tao, Y., Zhu, J., Small, D. S., Basner, M., Norton, L., Novak, C., Dine, J., Rosen, I. M., \& Volpp, K. G. (2014). Impact of protected sleep period for internal medicine interns on overnight call on depression, burnout, and empathy. Journal of Graduate Medical Education, 6(2), 256-263. https://doi.org/10.4300/JGME-D-1300241.1

Sinsky, C. A., Dyrbye, L. N., West, C. P., Satele, D., Tutty, M., \& Shanafelt, T. D. (2017). Professional satisfaction and the career plans of US physicians. Mayo Clinic Proceedings, 92(11), 1625-1635. https://doi.org/10.1016/j.mayocp.201 7.08.017

Siu, C. F. Y., Yuen, S. K., \& Cheung, A. (2012). Burnout among public doctors in Hong Kong: Cross-sectional survey. Hong Kong Medical Journal, 18(3), 186-192. https://www.hkmj.org/abstracts/v18n 3/186.htm 


\begin{tabular}{|l|l|l}
\hline 1 & $\begin{array}{l}\text { HEALTHY } \\
\text { POPULATIONS } \\
\text { JOURNAL }\end{array}$ \\
\hline
\end{tabular}

Slade, S. (2014). IMGs in Canada: Data and the changing gestalt [PowerPoint slides]. Royal College of Physicians and Surgeons of Canada. https://www.royalcollege.ca/rcsite/do cuments/health-policy/imgs-in-canadafeb-2014-steve-slade.pdf

Soler, J. K., Yaman, H., Esteva, M., Dobbs, F., Asenova, R. S., Katic, M., Ozvacic, Z., Desgranges, J. P., Moreau, A., Lionis, C., Kotanyi, P., Carelli, F., Nowak, P. R., de Aguiar Sa Azeredo, Z., Marklund, E., Churchill, D., Ungan, M., \& European General Practice Research Network Burnout Study Group. (2008). Burnout in European family doctors: The EGPRN study. Family Practice, 25(4), 245-265. https://doi.org/10.1093/fampra/cmn0 38

The Canadian Medical Protective Association. (2018a). Healthier physicians: An investment in safe medical care. https://www.cmpa-acpm.ca/staticassets/pdf/about/annualmeeting/18_healthier_physicians_backg rounder-e.pdf

The Canadian Medical Protective Association. (2018b, August 22). Canadian Medical Protective Association calls for systemwide change to improve physician wellness [Press release]. https://www.cmpaacpm.ca/en/connect/mediaroom/2018/canadian-medicalprotective-association-calls-for-systemwide-change-to-improve-physicianwellness

Vo, E. (2019, August 30). Why do you discount cash flows? The Hartford. https://sba.thehartford.com/finance/ca sh-flow/discounting-cash-flows/

World Health Organization. (2010). Monitoring the building blocks of health systems: $A$ handbook of indicators and their measurement strategies. https://www.who.int/workforceallianc e/knowledge/toolkit/26/en/ 